\title{
94 Sportverletzungen und Schmerzabwehr
}

\author{
(c) Springer-Verlag GmbH Deutschland, ein Teil von Springer Nature 2018 \\ D. Mathias, Fit und gesund von 1 bis Hundert \\ https://doi.org/10.1007/978-3-662-56307-6_94
}

Drohende oder bereits bestehende Gewebeschäden werden durch spezielle Sinnesfühler für Schmerzen erkannt. Diese sog. Nozizeptoren finden sich als Enden der dünnen A- und C-Nervenfasern in allen Organen außer im Zentralnervensystem. Über diese Fasern gelangen Schmerzreize zunächst zu den Wide-dynamic-range-Neuronen im Hinterhorn des Rückenmarks und werden von dort über Zwischenneurone zum Gehirn weitergeleitet. Hier erst entsteht der Sinneseindruck "Schmerz«. Bleiben Schmerzen unbehandelt, geraten die WDRNeurone unter Dauerbeschuss ständiger Impulse und die beschriebenen Abläufe schaukeln sich auf. Die Leitung von Schmerzsignalen wird durch Neubildung von Rezeptoren und Ionenkanälen vervielfacht. Auch zuvor unterschwellige Aktionspotenziale werden jetzt registriert, sogar Spontanentladungen dieser Nervenzellen ohne Schmerzmeldungen der betroffenen Nozizeptoren sind möglich. Aus allem resultiert eine verstärkte Schmerzwahrnehmung.

Glücklicherweise verfügt der Mensch über eine sehr wirksame körpereigene Schmerzabwehr.

So werden vom Hirnstamm ausgehend besondere Botenstoffe und Opioide ausgeschüttet, die die Aktionen der den Schmerz weiterleitenden WDR-Neurone deutlich einschränken. Eine wichtige Auf- gabe bei der Schmerzabwehr kommt auch jenen Nervenzellen zu, die in diesem Geschehen eine blockierende Funktion haben. Sie sind die Gegenspieler der Schmerzneurone und sind zwischen ihnen angeordnet. Bei jedem akuten Schmerz werden sie ebenfalls aktiviert und damit Überreaktionen in den schmerzverarbeitenden Systemen heruntergeregelt.

Körperliche Aktivitäten fördern die Funktion der schmerzhemmenden Nervenzellen.

Bei lang anhaltenden Reizungen jedoch versagen leider die Mechanismen der Schmerzbegrenzung häufig. Dann droht die Gefahr der Schmerzchronifizierung mit Ausbildung eines Schmerzgedächtnisses. Hiervon sind am ehesten Menschen mit Kopfschmerzen und Rückenproblemen betroffen. Die schnelle und dauerhafte Schmerzbekämpfung ist deshalb sehr wichtig.

Sportverletzungen erfordern also eine große Sorgfalt in ihrer Behandlung. Weil aktive Bewegungen die Schmerzbekämpfung effektiv unterstützen, sollte ein Aufbautraining so früh wie möglich beginnen. Allerdings muss dabei übertriebener Ehrgeiz vermieden werden, denn verbleibende Restschmerzen können leicht falsche Bewegungsmuster bahnen, die oft muskuläre Dysbalancen und erneute Schmerzen nach sich ziehen. 